\title{
EchoGéo
}

16 | 2011

Échos de Turquie

\section{Géographie des interfaces. Une nouvelle vision des territoires}

Compte rendu de lecture

Marie Redon

\section{OpenEdition}

Journals

\section{Édition électronique}

URL : https://journals.openedition.org/echogeo/12443

DOI : $10.4000 /$ echogeo.12443

ISSN : 1963-1197

Éditeur

Pôle de recherche pour l'organisation et la diffusion de l'information géographique (CNRS UMR 8586)

\section{Référence électronique}

Marie Redon, «Géographie des interfaces. Une nouvelle vision des territoires », EchoGéo [En ligne], 16। 2011, mis en ligne le 04 juillet 2011, consulté le 25 août 2021. URL : http://journals.openedition.org/ echogeo/12443; DOI : https://doi.org/10.4000/echogeo.12443

Ce document a été généré automatiquement le 25 août 2021.

EchoGéo est mis à disposition selon les termes de la licence Creative Commons Attribution - Pas d'Utilisation Commerciale - Pas de Modification 4.0 International (CC BY-NC-ND) 


\title{
Géographie des interfaces. Une nouvelle vision des territoires
}

\author{
Compte rendu de lecture
}

Marie Redon

1 Littéralement, une interface est une surface de séparation entre deux états distincts de la matière. Le terme, ayant cheminé de la physique à la biologie, et aussi désormais d'usage répandu en informatique. En géographie, l'usage du mot s'est développé dans les années 1980 et peut être défini comme un espace permettant la mise en relation de deux espaces/territoires différents, influencé par des échanges entre l'un et l'autre, et se distinguant par là-même des deux espaces contigus. Intégrant la notion de contact ("plan ou ligne de contact entre deux systèmes ou deux ensembles distincts », R. Brunet, 1993), l'interface met l'accent sur les interactions spatiales et se trouve même définie comme une forme d'« interspatialité » (J. Lévy et M. Mussault, 2003).

2 L'ouvrage Géographie des interfaces. Une nouvelle vision des territoires fait suite à un article publié dans la revue L'Espace géographique par le Groupe Interfaces, en 2008. Alors que cet article explorait la notion d'interface et en proposait une grille de lecture, il s'agit ici de mettre en pratique la méthodologie alors élaborée en la confrontant à des cas concrets. L'avant-propos intitulé « complexité du Système Interface » reprend donc des éléments de l'article de 2008 et introduit une série d'études de cas au préalable synthétisés dans une "grille de lecture des interfaces " qui part de son identification (origine du différentiel sur lequel elle émerge), en précise les fonctions (échange, régulation, mais aussi transfert) avant de décrire les différents mécanismes internes à l'interface (attracteur, sélecteur, adaptateur et commutateur). Cette grille de lecture aboutit à un "score d'interfacité » et un "degré d'interfacité » qui permettent une typologie des interfaces selon ce gradient: fonction de continuité, interfaces « significatives », interfaces puissantes.

3 Une fois ce préalable posé, les huit textes qui composent le cœur du livre présentent divers exemples de systèmes localisés, les interfaces pouvant être de type horizontal (articulation entre espaces de même niveau d'organisation) ou vertical (niveaux d'organisation différents). L'ouvrage est divisé en quatre parties, chacune composée de 
deux contributions. Le découpage thématique (temporalités, accessibilités, mondialisation) aurait peut-être mérité d'être étayé par une petite introduction explicitant les relations entre les types d'interfaces analysés au sein de chaque partie.

Ainsi, la première partie est consacrée aux interfaces comme "espaces en mutation rapide ». Le texte de J.P. Ferrier, se présentant comme une épistémologie exploratoire partant du postulat que l'interface nature-culture « doit être reconnue comme l'un des opérateurs géographiques ( $\left.G^{*}\right)$ qui gère la mesure dans les territoires ", y est juxtaposé à une évaluation du risque d'incendie de forêt en Provence (C. Lampin-Maillet) via l'interface habitat-forêt. A partir d'une typologie croisant des critères relevant des structures de l'habitat et des structures de la végétation, une cartographie des interfaces habitats-forêt est dressée. Celle-ci permet à son tour d'établir une carte de l'indice de risque d'incendie qui s'avère maximal en cas de zones d'habitat isolé avec une forte agrégation de végétation et minimal dans le cas contraire. L'intérêt de cette approche serait de produire un décentrement des acteurs par rapport à leurs territoires de compétence et d'action en favorisant des processus transversaux de décision. La proposition est stimulante, mais en s'éloignant du texte précédent, par lequel l'auteur propose de nouvelles conceptions de l'interface nature-culture d'abord dans une logique binaire (avec un recours à un test écologique et à un test démocratique) puis ternaire (« nature | culture / interface » en tant que « méta-triade conceptuelle »), elle ajoute une strate supplémentaire à une construction intellectuelle déjà touffue, sans nécessairement la rendre plus accessible. Si ce complexe article inaugural s'ouvre «vers un nouveau programme géographique» prônant une géographie active portant sur les questions "sensibles» des territoires, le lecteur regrettera sans doute de ne pas en voir d'application systématique.

5 La deuxième partie montre l'existence de cycles de vie des interfaces (genèse, montée en puissance, optimum, résilience ou déclin, voire disparition) et leur irréversibilité : parce qu'elles répondent à un besoin, elles deviennent aussi créatrices de besoin. $\mathrm{P}$. Allard propose ainsi une approche historique du Rhône longitudinal (route commerciale depuis l'Antiquité) puis du Rhône transversal (obstacle physique mais aussi administratif et politique); le cas servait déjà d'illustration à l'article de 2008. Après l'interface fluviale, l'interface terre-mer, nouvelle « donne » de la littoralisation, est abordée par I. Morr à partir de l'exemple de Cagnes-sur-Mer et de Cros de Cagnes (Provence). Elle pointe l'apparition de risques, en termes de gestion et d'aménagement du littoral, dus au décalage temporel entre une "culture de la côte", en voie de disparition sous des usages et des références terriennes et urbaines, et une interface terre-mer toujours basée sur les mêmes variables physiques.

6 La troisième partie traite de transports et d'accessibilité, considérant les interfaces comme des "pôles d'échanges ». La première étude de cas (L. Chapelon), centrée sur la gare de Nîmes, fait des interfaces un outil déterminant pour éclairer les politiques d'aménagement: l'intermodalité permettrait de remédier au problème de saturation routière en intégrant différents niveaux d'organisation dans une chaine complète de déplacements. La contribution suivante propose une analyse de la zone située entre Marseille et Gênes; l'étude multiscalaire et multisituée des réseaux ferrés (gares de Vintimille, Monaco, Nice et Breil-sur-Roya) menée par G. Fusco montre que cet espace métropolitain transfrontalier présente des faiblesses quant aux interfaces ferroviaires, détectables par l'identification de lieux stratégiques parfois délaissés que sont les nœuds (gares). Là aussi, une présentation commune de l'articulation entre les notions 
d'interface, de pôles, de nœuds et d'intermodalité manque à la compréhension et à la lisibilité d'ensemble.

7 La dernière partie de l'ouvrage traite de deux exemples d'interfaces de la mondialisation : le Tourisme de réunion et de congrès international (TRCI) et la santé. S. Christofle livre une analyse des interfaces immatérielles (réunion-activité congressuelle) et matérielles (palais des congrès), à la croisée entre le monde professionnel/entrepreneurial et celui du tourisme et des loisirs. Une entrée par les interfaces permettrait ainsi de mieux comprendre la volonté des décideurs politiques et des acteurs socio-économiques d'attirer ces flux afin d'intégrer la ville dans la compétition interurbaine. Dans l'article "Santé et interface», S. Pérez examine trois types d'interfaces sanitaires: reposant sur des opérations de coopérations transfrontalières, notamment européenne (télémédecine entre la Suisse et l'Allemagne, hôpital transfrontalier franco-espagnol, etc.), relevant du "tourisme sanitaire " (Turquie spécialisée en ophtalmologie, Thaïlande attirant les Laotiens, etc.) puis, enfin, les opérations de « crise » de grandes envergures. Ces interfaces reflèteraient la volonté des acteurs de "rompre avec l'absurde » en rationalisant les pratiques. Là encore, le lecteur pourra regretter que le fil politique sous-tendant les deux textes (à qui l'interfacité profite-t-elle ?) ne soit un peu déroulé, notamment à partir d'exemples.

Cette Géographie des interfaces offre donc un bel ensemble de monographies montrant la variété des champs et des échelles d'application de la notion. Mais si le propos initial était de faire émerger de "l'inédit » en considérant les interfaces comme des systèmes socio-spatiaux à part entière, produisant autre chose que la simple addition d'éléments en interaction, le lecteur aurait pu attendre, précisément, un peu plus d'interfacité entre ces études d'interface.

\section{BIBLIOGRAPHIE}

Lampin-Maillet C., Pérez S., Ferrier J-P., Allard P., (coord.), 2010. Géographie des interfaces. Une nouvelle vison des territoires. Editions Quae, $165 \mathrm{p}$.

\section{AUTEUR}

\section{MARIE REDON}

Marie Redon est maître de conférences à l'université Paris VIII. mredon@yahoo.com 